\title{
Ks1, an epithelial cell-specific gene, responds to early signals of head formation in Hydra
}

\author{
Ruth Weinziger, Luis M. Salgado*, Charles N. David and Thomas C. G. Bosch ${ }^{\dagger}$ \\ Zoological Institute, University Munich, Luisenstrasse 14, 80333 Munich, FRG \\ *Present address: Instituto de Biotecnologia - UNAM, Departmento de Biochimica, Apartado 510-3, 62271 Cuarnavaca, Mexico \\ tAuthor for correspondence
}

\section{SUMMARY}

As a molecular marker for head specification in Hydra, we have cloned an epithelial cell-specific gene which responds to early signals of head formation. The gene, designated $k s 1$, encodes a 217-amino acid protein lacking significant sequence similarity to any known protein. KS1 contains a $\mathrm{N}$-terminal signal sequence and is rich in charged residues which are clustered in several domains. $k s 1$ is expressed in tentacle-specific epithelial cells (battery cells) as well as in a small fraction of ectodermal epithelial cells in the gastric region subjacent to the tentacles. Treatment with the protein kinase $\mathrm{C}$ activator 12- $\mathrm{O}$-tetradecanoylphorbol-13acetate (TPA) causes a rapid increase in the level of $k s 1$
mRNA in head-specific epithelial cells and also induces ectopic $k s 1$ expression in cells of the gastric region. Sequence elements in the 5 -flanking region of $k s 1$ that are related to TPA-responsive elements may mediate the TPA inducibility of $k s 1$ expression. The pattern of expression of $k s 1$ suggests that a ligand-activated diacylglycerol second messenger system is involved in head-specific differentiation.

Key words: hydra, regeneration, pattern formation, protein kinase $\mathrm{C}$, phosphatidylinositol, TPA, TRE

\section{INTRODUCTION}

Knowledge of the mechanisms of position-dependent differentiation of specific cell types is central to understanding how embryos are formed. 25 years ago, Lewis Wolpert (1969) introduced the concept of positional information and proposed that cells within a developing system are informed of their position with respect to one or more reference points. The cells then acquire a positional value and interpret their positional value by differentiating in a particular way. One example of pattern formation considered in detail by Wolpert was development and regeneration in Hydra (Wolpert, 1969; Wolpert et al., 1974).

Development in hydra consists mainly of head and foot formation from tissue of the body column (see Bode and Bode, 1984). When head and/or foot are removed, they regenerate within two to three days. A head always regenerates at the apical end and a foot regenerates at the basal end. These and other observations have produced strong evidence for the existence of positionally restricted 'morphogenetic' molecules in hydra (see Bode and Bode, 1984; Summerbell et al., 1991). The naturally occurring morphogens have not yet been identified. Considerable progress, however, has been made recently towards the molecular analysis of axial patterning in hydra.

Several homeodomain genes have been identified as candidates for specification of positional information (for review see Shenk and Steele, 1993). The expression pattern of one of them, cnox-2, is consistent with a role in axial pattern formation (Shenk et al., 1993). Furthermore, several genes have been isolated that become activated in hydra in spatial and temporal patterns (Kurz et al., 1991; Lopez de Haro et al., 1994).

The signal transduction system used in axial patterning in hydra has recently begun to be examined. Treatment with diacylglycerol (DAG) or 12-O-tetradecanoylphorbol-13-acetate (TPA) was found to induce ectopic heads in the body column (reviewed in Müller, 1993). Prolonged exposure of polyps to lithium causes appearance of ectopic feet along the body column (Hassel et al., 1993). Coadministration of DAG with arachidonic acid, a subordinate signaling molecule in the phosphatidylinositol-protein kinase C (PI-PKC) system, causes a strong increase in the head formation potential (Müller et al., 1993). DAG and TPA are known to activate PKC (Nishizuka, 1986) while lithium blocks several enzymes in the PI-PKC system (Berridge et al., 1989). Thus, it appears likely that a PKC-mediated intracellular pathway initiated by a ligandreceptor interaction on the cell surface mediates head specification in hydra.

Candidate genes responding to such a signaling system should be (i) expressed in head-specific cells, (ii) induced by TPA, and (iii) contain TPA-responsive elements in their promoter. Here we report the cloning and characterization of a gene, $k s 1$, which fulfills these criteria and thus supports the view that a ligand-activated PI-PKC system is involved in the conversion of hydra gastric tissue to head tissue. 


\section{MATERIAL AND METHODS}

\section{Animals}

Polyps of Hydra magnipapillata (strain sf-1) and Hydra vulgaris were used in this study. Polyps were cultured according to standard procedure (Lenhoff and Brown, 1970) at $18^{\circ} \mathrm{C}$.

\section{TPA treatment}

TPA was dissolved in acetone at $10 \mathrm{mM}$ and stored at $-20^{\circ} \mathrm{C}$. Polyps were incubated in TPA at a final concentration of 30-50 $\mathrm{nM}$ (in hydra medium) for 20 minutes at $18^{\circ} \mathrm{C}$. After treatment the animals were transferred to hydra medium and cultured at $18^{\circ} \mathrm{C}$ until they were used.

\section{cDNA library construction and differential screening}

$1 \times 10^{5}$ amplified recombinants of a oligo(dT)-primed epithelial cellspecific cDNA library (Lopez de Haro et al., 1994) were screened with ${ }^{32} \mathrm{P}$-labeled cDNA made from head and gastric tissue of epithelial polyps by the random primer labeling procedure. 19 clones were picked as head specific; 17 of these clones encoded a single gene, designated $k s l$. The remaining 2 clones encoded different genes and are described elsewhere (Lopez de Haro et al., 1994). Since none of the $17 k s 1$ cDNA clones was full length, one of the clones (cLK7) was used to rescreen a $H$. vulgaris cDNA library. The cDNA library in lambda ZAPII was prepared commercially (Stratagene) from Hydra vulgaris whole polyp poly(A)+ RNA and was generously provided by Drs Michael Sarras (University of Kansas Medical Center) and Hans Bode (University of California, Irvine). Rescreening resulted in the isolation of a number of additional clones, one of which was cLK741. Sequence analysis of cLK7-41 indicated the presence of an open reading frame beginning at the $5^{\prime}$ most ATG triplet. cLK7-41, however, did not encode $3^{\prime}$ sequences present in the initially isolated $k s 1$ clones. To complete the sequence and to confirm the region of overlap between cLK7-41 and the initial cDNA clones, inverse PCR was carried out as described below.

\section{Isolation of genomic sequences by inverse PCR}

To obtain the full-length sequence of $k s l$ as well as $5^{\prime}$-flanking sequences, inverse PCR was carried out as described (Gellner et al., 1992) using two 20-nucleotide primers oriented such that primerextension proceeded outward from the known sequence. Cleavage of $H$. vulgaris genomic DNA with HindIII resulted in a $3 \mathrm{~kb}$ fragment, which in Southern blots strongly hybridizes with cLK7-41. For inverse PCR, therefore, HindIII-digested and ligated DNA was used as template DNA. Oligonucleotide primers were synthesized complementary to nucleotides $84-103$ and identical to nucleotides 297319 (see Fig. 2). The expected $1.7 \mathrm{~kb}$ inverse PCR product was obtained and cloned into pBS- for further analysis. The relationship of this PCR-derived genomic clone, gIPCR1, to the cDNA clones is shown in Fig. 1.

\section{Molecular techniques}

Nucleic acid isolation, sequence analysis and RNA blot analysis were carried out following standard procedures (Sambrook et al., 1989). DNA sequences were analysed using the Hibio DNASIS/PROSIS program (Hitachi). All polymerase chain reactions (PCR) were carried out using the buffer conditions and Taq polymerase supplied by the manufacturer (Amersham). The $5^{\prime}$ boundary of the $k s 1$ transcript was determined by primer-extension following standard procedures. The 20-nucleotide IPCR5 primer was $5^{\prime}$ end-labeled, mixed with $9 \mu \mathrm{g}$ of poly(A)+ RNA from head tissue, denatured and allowed to hybridize at $56^{\circ} \mathrm{C}$ for 16 hours as described previously (Gellner et al., 1992). After primer elongation and RNase A treatment, the primer extension products were separated by $8.3 \mathrm{M}$ urea/6\% PAGE and visualized by autoradiography. EMBL database accession number for $k s 1, \mathrm{X} 78596$.

\section{In situ hybridization on macerates and whole mounts}

In situ hybridization on whole mounts and macerates was carried out with digoxigenin-labeled DNA probes as described by Kurz et al. (1991) with the following modifications. Postfixed whole mounts were washed for 15 minutes in PBT, then for 20 minutes in 1/1 hybridization solution (HS)/PBT at room temperature and for 20 minutes in $\mathrm{HS}$ at room temperature. Afterwards whole mounts were prehybridized for 20 minutes in $\mathrm{HS}$ at $45^{\circ} \mathrm{C}$ and hybridized as described.

\section{RESULTS}

\section{Isolation and nucleotide sequence of $k s \mathbf{1}$}

We used a differential cDNA screening approach to isolate hydra genes expressed in head but not in gastric tissue. We focused our attention on epithelial cells, since this is the cell type responsible for morphogenesis in hydra. We isolated a number of clones encoding a tentacle-specific cDNA, which we have designated $k s l$. Since none of the cDNA clones was full length, we isolated additional clones from a cDNA library and a genomic clone by inverse PCR (see Materials and Methods) to complete the sequence.

The nucleotide sequence of the $k s 1$ gene was obtained from the combined results of the $H$. vulgaris cDNA and genomic clones shown in Fig. 1. The $k s l$ sequence contains an open reading frame of $651 \mathrm{bp}$ (Fig. 2). At the $5^{\prime}$ end, 392 nucleotides precede an ATG triplet. 15 bp upstream of the first in-frame methionine codon, there is an in-frame termination codon indicating that the first ATG triplet is indeed the initiator. The length of the transcript predicted from the combined length of the cDNA clones (906 bp) corresponds well to $0.9 \mathrm{~kb}$ mRNA detected on northern blots (see below) indicating that the fulllength cDNA has been identified. Conceptional translation of the open reading frame predicts a 217 amino acid protein with a relative molecular mass of $25.4 \times 10^{3}$.

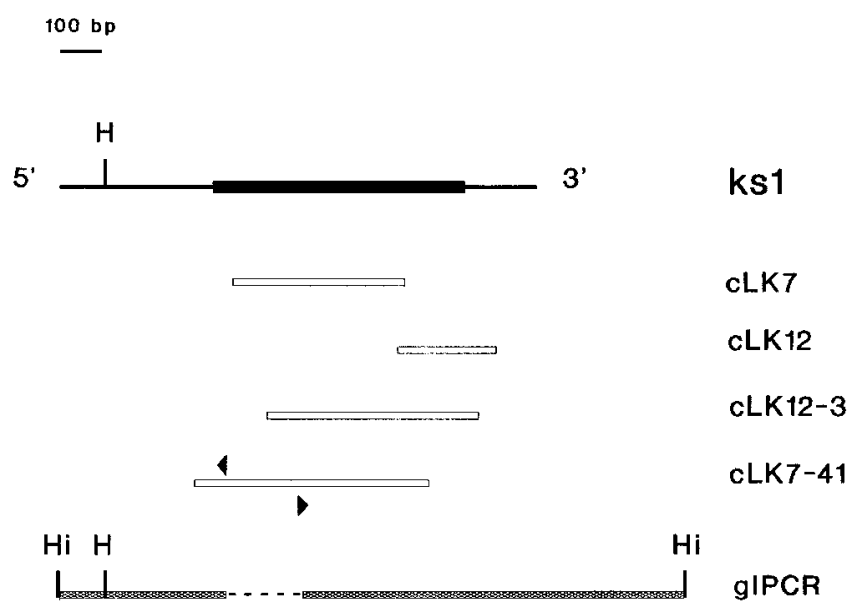

Fig. 1. Schematic representation of $k s 1$ clones and the $k s 1$ gene. Clones cLK7 and cLK12 were isolated by differential screening of a H. magnipapillata cDNA library. Subclone cLK12-3 was isolated from the same library by rescreening with cLK12. The nearly fulllength cDNA clone cLK7-41 was isolated from a $H$. vulgaris $\mathrm{cDNA}$ library by rescreening with cDNA clone cLK7. The cDNA clones are represented by open bars. The genomic region amplified by inverse PCR (gIPCR) from H. vulgaris genomic DNA is represented as hatched bars. Filled arrowheads show position of primers used in inverse PCR. The solid bar in the $k s 1$ gene represents the coding region, the thin line indicates untranslated regions. H, HincII; Hi, HindIII. 


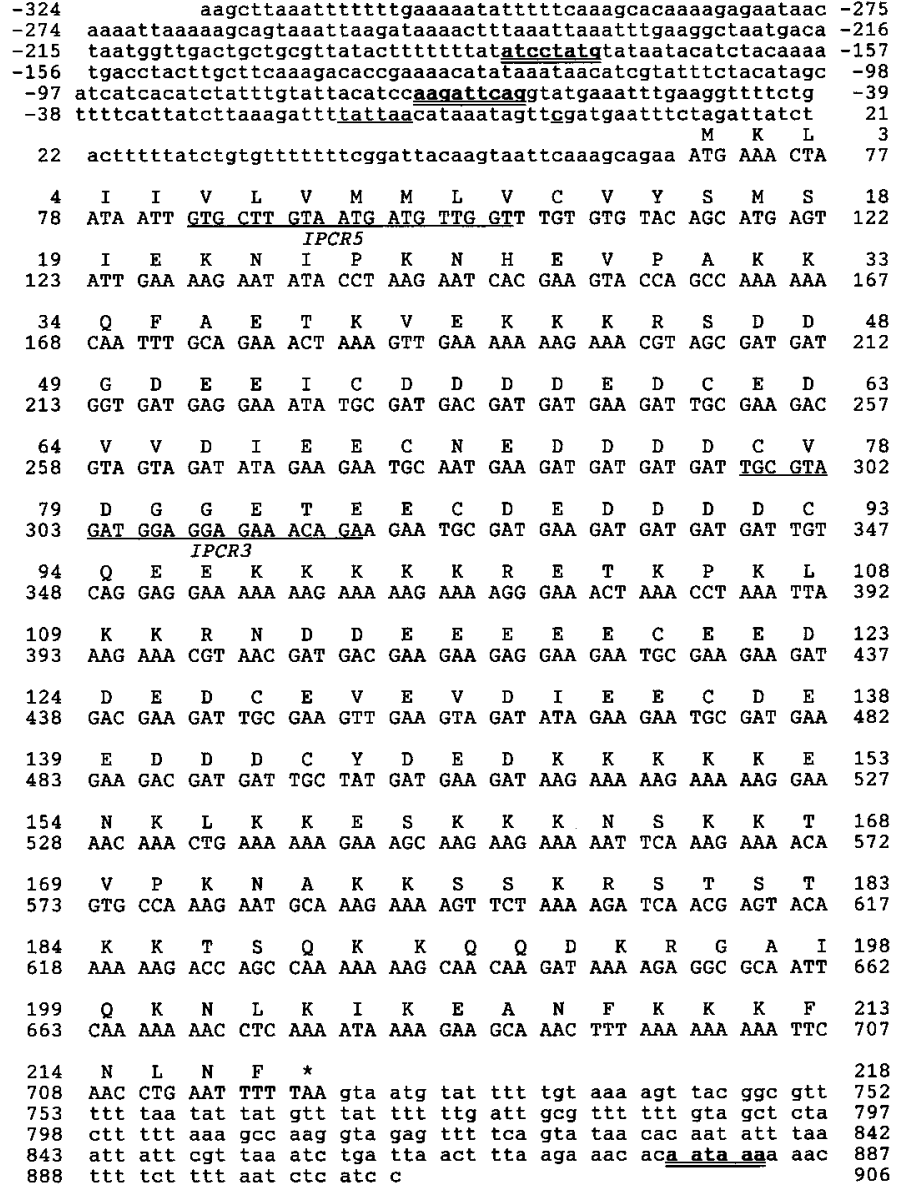

Fig. 2. Sequence of the $k s 1$ gene. The sequence was obtained from the $H$. vulgaris cDNA and genomic clones shown in Fig 1 . The complete sequence of the $k s l$ gene, the amino acid sequence of the single large open reading frame, as well as 580 nucleotides of flanking sequence are presented. Double underlines indicate putative regulatory elements (see Fig. 3 for details). Single underlines indicate primers used in inverse PCR.

The transcription initiation site was determined by primer extension using the IPCR5 primer complementary to nucleotide $84-103$ of $k s l$. The labeled primer was hybridized to RNA from head tissue and extended by reverse transcriptase. The single extension product indicated that transcription initiates 68 bases upstream of the translational initiation codon (data not shown).

To identify sequences that potentially control $k s l$ expression, we examined the $5^{\prime}$ flanking sequence for potential transcription factor binding sites. Comparison of this region with promoters of other genes revealed several segments of sequence conservation (Fig. 3). The sequence TATTAA (single underline in Fig. 3A) 17 bp upstream of the transcription start site is similar to TATA-like sequences in many eukaryotic promoters. At positions -62 to -70 the $k s l$ gene contains the sequence $5^{\prime}$-AAGATTCAG-3' (double underlined in Fig. 3A), which is similar to the TPA-responsive element (TRE, consensus sequence 5'-TGAGTCAG-3', Angel et al., 1987). A sequence comparison between TREs in the control regions of TPA-inducible genes and the putative TRE in $k s l$ is shown in Fig. 3B. The short conserved TRE sequence serves as the binding site for the transcription factor AP1 and is the only element required for TPA induction in a number of genes (Angel et al., 1987). At position -175 to -182 , there is a sequence related to the binding site for the transcription factor

\begin{abstract}
A

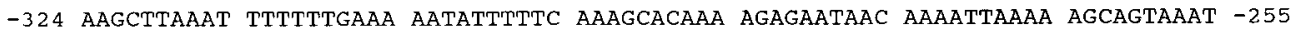

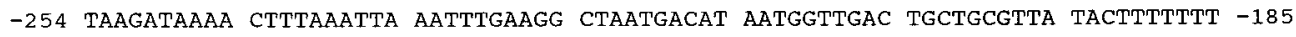
-184 AT ATCCTATg TATAATACAT CTACAAAATG ACCTACTTGC TTCAAAGACA CCGAaAacAT ATAAATAACA - 115 -114 TCGTATTTCT ACATAGCATC ATCACATCTA TTTGTATTAC ATCCAAGATT CAgGTATGAa ATTTGAAGGT -45 -44 TTTCTGTTTT CATTATCTTA AagATTTTAT TAACATAAAT AGTTCGATGA ATTTCTAGAT TATCtActTT 26 $+1$

27 TTATTAGACT GTGTTTTTTT CGgATtacAa gTAATTCAAA GCAGAAATg
\end{abstract} C

\section{B}

TPA inducible gene

TRE $^{1}$

\begin{tabular}{|c|c|c|c|c|}
\hline Hydra ksI & A & $\mathrm{A} A \mathrm{G}$ & $\mathrm{T}$ & $T \subset A G$ \\
\hline Human collagenase & & $T \in A$ & $G$ & T C A G \\
\hline Rat stromelysin & & $\mathrm{T} \in \mathrm{A}$ & $G$ & $T C A G$ \\
\hline Human MTIIA & $\mathrm{G}$ & $T \in A$ & c & T C A G \\
\hline Interleukin 2 & $\mathbf{T}$ & $\mathrm{T}|\mathrm{C}| \mathrm{A}$ & $G$ & $\mathrm{~T} C \mathrm{~A} G$ \\
\hline
\end{tabular}

1 For references see Angel et al. (1987)
Fig. 3. Analysis of the $5^{\prime}$ flanking sequence of Hydra ks-1. (A) Nucleotide sequence of the $5^{\prime}$ flanking region of the $k s-1$ gene. Only the coding-strand sequence, numbered from the transcription start site (indicated by an asterisk), is shown. The putative binding sites for transcription factors USF/MLTF (MTE) and AP1 (TRE) are indicated by double underlines. A single underline shows the putative TATA sequence. (B) Sequence comparison of putative TREs in the control regions of TPAinducible genes (Angel et al., 1987) and Hydra ks1. Sequences are aligned with respect to the invariant TCAG tetranucleotide stretch. Conserved nucleotides are enclosed in boxes. (C) Sequence comparison of the MTE sequence in the $5^{\prime}$ flanking

region of TPA-inducible human heme oxygenase gene and hydra $k s 1$. The two genes are aligned with respect to the three nucleotides (CNNNTG) shown to be crucial for protein binding (Muraosa Y. and S. Shibahara, 1993). Conserved nucleotides are enclosed in boxes. 
MLTF/USF in the TPA-inducible heme oxygenase gene (Muraosa and Shibahara, 1993). As shown below, hydra $k s l$ is responsive to TPA (see Fig. 9). Thus, it is the first TPA responsive gene known to contain both TRE and MLTF/USF sequences in the $5^{\prime}$ flanking region. At the $3^{\prime}$ terminus, a consensus polyadenylation sequence, AATAAA, is located at position 878-883 (Fig. 2).

\section{The $k s-1$ protein is composed of several structural domains}

A search of various protein data banks with the KS1 amino acid sequence revealed no homology between KS1 and any other protein. KS1, however, contains several interesting structural features (Fig. 4). Hydropathy analysis of KS1 predicted a strongly hydrophilic character (Fig. 4A). The N-terminal 15 residues are strongly hydrophobic, indicative of a signal sequence for translocation across the endoplasmic reticulum. An acceptable cleavage site for a signal peptidase is present between Ser16 and Met17 (von Heijne, 1983). It seems likely, therefore, that KS1 encodes a secreted protein. No potential Nlinked glycosylation sites are present in the KS1 sequence.

One striking feature of the predicted KS1 protein is the large number of charged residues that are clustered into highly acidic and basic domains (Fig. 4B). Beginning at residue 32, 7 out of the next 15 amino acids are lysine and arginine. This basic domain (B1 in Fig. 4B) is followed by a acidic domain (A1 in Fig. 4B) consisting of $66 \%$ aspartic acid and glutamic acid residues. This acidic domain is immediately followed by a 16 amino acid basic region (B2), including a stretch of 6 consecutive lysine and arginine residues. The 35 amino acid domain A2 following this basic domain shows remarkable amino acid similarity to the first acidic domain A1. The carboxy terminal domain (B3) contains $42 \%$ lysine or arginine residues.

Analysis of the deduced amino acid sequence revealed two regions of internal sequence similarity comprising residues 31-96 and 97-146. The repeated sequences are aligned in Fig. 4C. Structuralprediction algorithms (ChouFasman) predict a helix-turnhelix structure for these repeats.

\section{Position-dependent expression of $k \mathbf{s} \mathbf{1}$}

To determine the expression pattern of $k s l$, we analyzed northern blots of RNA from head, upper one third and lower two thirds of $H$. vulgaris polyps (Fig. 5). In head tissue a single, abundant $0.9 \mathrm{~kb}$ transcript was detected. The same transcript was also found in the upper third of the body column, although at a much lower level (lane 2 in Fig. 5). No ks 1 transcripts were detected in the lower two thirds.
To determine the cell-type specificity of $k s l$ expression, we performed in situ hybridizations on macerated cells (Fig. 6). Fig. 6A shows that, in macerates hybridized with clone cLK12, tentacle-specific ectodermal epithelial cells (battery cells) were heavily labelled. The occurrence of $k s l$ transcripts in the upper third of the gastric region, which includes the 'tentacle formation zone' (Fig. 5, lane 2), suggests that there is a population of cells in this region not yet differentiated to tentaclespecific cells but already expressing $k s l$. To test this possibility, we performed in situ hybridization on macerates from the upper third of the gastric region. Fig. 6B shows that in this region $k s l$ hybridizes to a population of epithelial cells which are morphologically indistinguishable from gastric epithelial cells. Cell counts indicated that about $10 \%$ of the ectodermal epithelial cells in the 'tentacle formation zone' region are $k s 1$ positive. These cells appear to represent body column cells determined to differentiate to tentacle epithelial cells. Thus, $k s I$ expression appears to be initiated early in head specification.

\section{ks1 expression during head regeneration}

Activation of $k s l$ expression during head regeneration was studied by in situ hybridization on whole mounts (Fig. 7). Animals were decapitated directly below the tentacles and hybridized to $k s 1$ 1, 2 and 4 days later. $k s l$ expression could be detected in ectodermal epithelial cells by 2 days when tentacles were beginning to evaginate (Fig. 7C,D). When regenerating heads at this stage were viewed from above (Fig. 7D), staining could also be seen in the most apical region of the hypostome (arrow in Fig. 7D). In mature heads (Fig. 7E,F) staining was restricted to the tentacles: staining appeared to end abruptly at the base of the tentacle; no staining was found between the tentacles. In whole mounts, it was not possible to detect $k s 1$-positive cells in the upper third of the gastric region. Thus, the whole-mount in situ procedure appears to be too

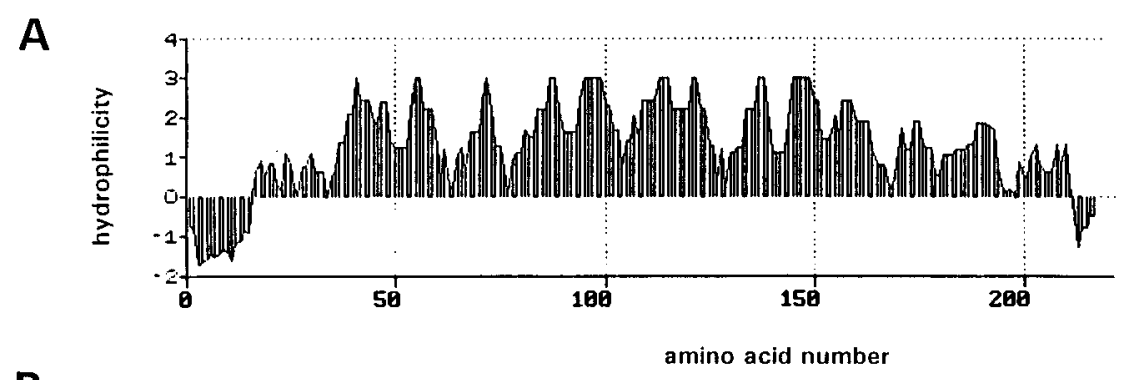

B

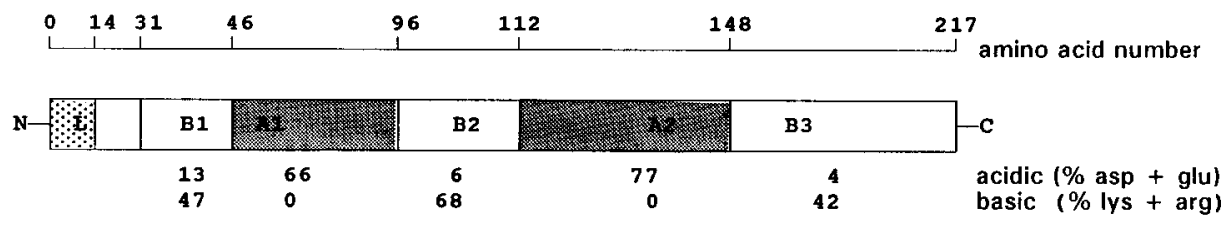

C

\begin{tabular}{|c|c|c|c|}
\hline & & B1 / B2 & $\mathrm{A} 1 / \mathrm{A} 2$ \\
\hline $\begin{array}{l}11 \mathrm{~A} 1 \\
2 \mathrm{~A} 2\end{array}$ & & $\begin{array}{l}\text { KKOFAETKVEKKKRS } \\
\text {..KKR...PKL...N }\end{array}$ & 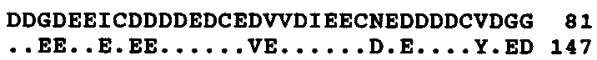 \\
\hline
\end{tabular}

Fig. 4. Analysis of the KS1 amino acid sequence. (A) Hydropathy plot of the predicted KS1 product. (B) Domain structure of KS1. Cross-hatched areas, acidic domain; open boxes, basic domains; stippled, signal peptide. (C) Internal sequence similarity in two stretches of KS1 sequence. Dots represent identical amino acids. 
1

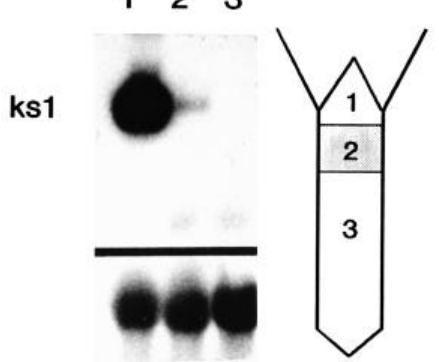

Fig. 5. Spatial expression pattern of $k s 1$ in hydra. $15 \mu \mathrm{g}$ of total RNA was isolated from the region indicated and hybridized with a ${ }^{32} \mathrm{P}$ labeled $k s 1 \mathrm{cDNA}$ probe. A ribosomal DNA fragment was used as control to demonstrate equal loading of RNA (Bosch et al., 1989).

insensitive to detect the $k s l$ expressing epithelial cells observed in macerates of the tentacle formation zone (Fig. 6B).

\section{ks1 expression in TPA-treated polyps}

Treatment of hydra with the PKC activators DAG or TPA results in appearance of ectopic head structures in the body column (Müller, 1989, 1993). To investigate whether TPA caused changes in the pattern of $k s l$ expression, we exposed decapitated polyps to TPA for 20 minutes and allowed heads to regenerate for 1,4 and 24 hours. Thereafter regenerating polyps were cut in the middle and the resulting upper and lower body column segments were assayed for $k s l$ expression using northern blotting. The results in Fig. 8 demonstrate that treatment with TPA alters $k s l$ expression significantly.

In the upper portions of TPA-treated polyps, the level of $k s l$ transcripts increased dramatically within 1 hour compared to untreated animals. In situ hybridization of macerated tissue from these animals indicated that there was no corresponding increase in the proportion of $k s 1$-expressing cells compared to untreated animals. Thus, the increase in $k s 1$ transcripts appears to represent an activation of transcription in $k s l$-expressing cells. In the lower portions of TPA-treated animals, there were no rapid changes in ks1 expression. There was, however, an increase in $k s l$ expression beginning 4 hours after TPA treatment. Cells in this region do not normally express $k s l$ (Fig. 5) and hence this expression represents ectopic activation of a positionally dependent gene.

The results in Fig. 8 also indicate that $k s 1$ transcription is activated during normal head regeneration. The increase in $k s l$ transcripts is detectable beginning by
4 hours of regeneration and thus is delayed compared to the rapid effect of TPA (see Discussion).

\section{DISCUSSION}

Patterning in hydra has been studied extensively at the tissue level using grafting techniques and monoclonal antibodies (for reviews see Bode and Bode, 1984; Müller, 1993). No probes have been available, however, for examining the patterning process at the molecular level. In this report, we present the molecular cloning of such a probe.

\section{The head-specific $k s 1$ gene encodes a novel protein}

The sequence of the $k s l$ gene product has no similarity to other proteins in various databases. KS1 is predicted to contain a
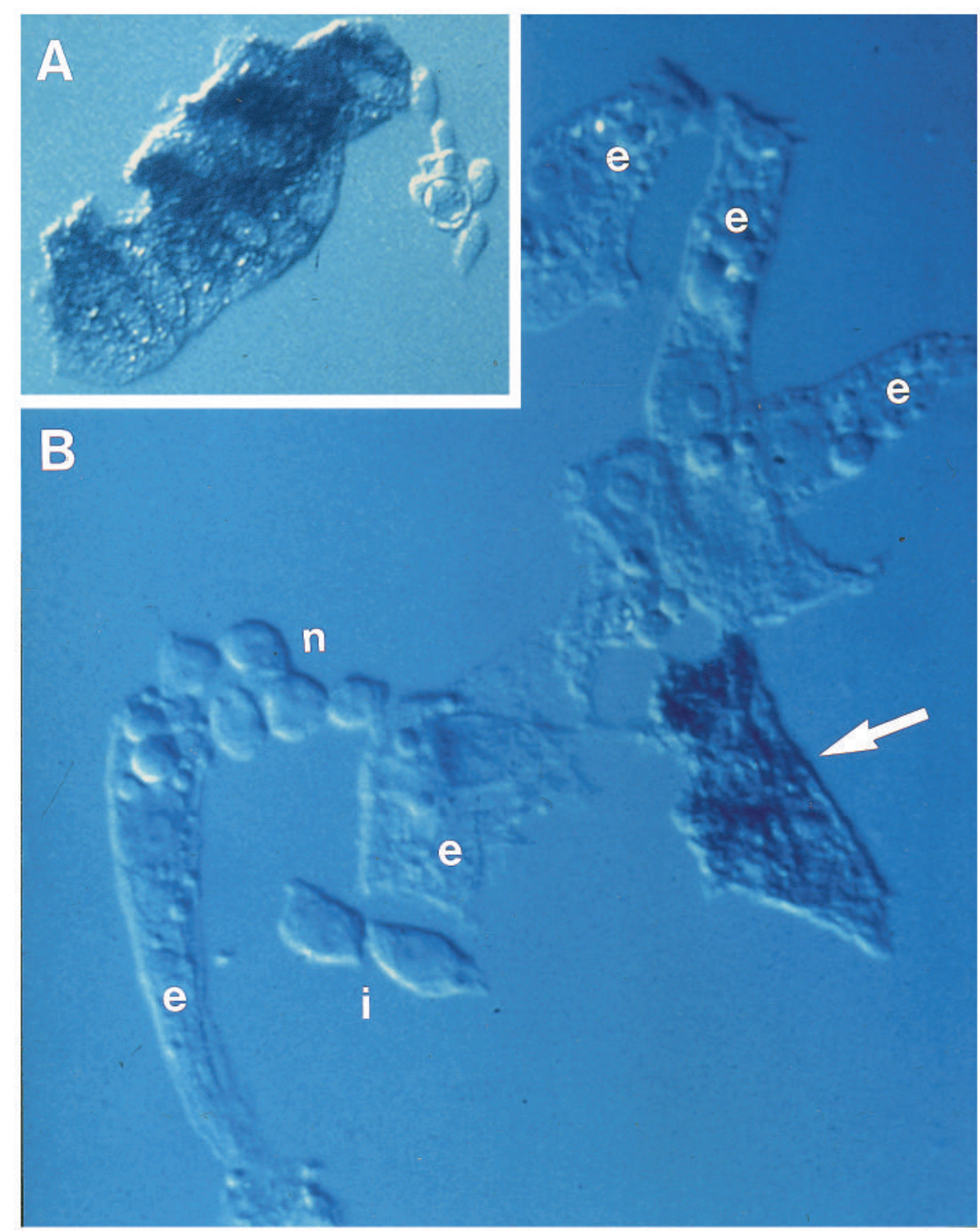

Fig. 6. Expression of $k s 1$ in macerated cells. (A) Macerates from whole polyps showing heavily stained tentacle-specific epithelial cell. (B) Macerates from the upper gastric region (region 2 in Fig. 5) showing $k s l$-expressing ectodermal epithelial cell (arrow). e, epithelial cell; i, interstitial cell; $\mathrm{n}$, nematoblasts. $k s 1$ clone cLK7 labeled with digoxigenin-dUTP was used as probe. 
A
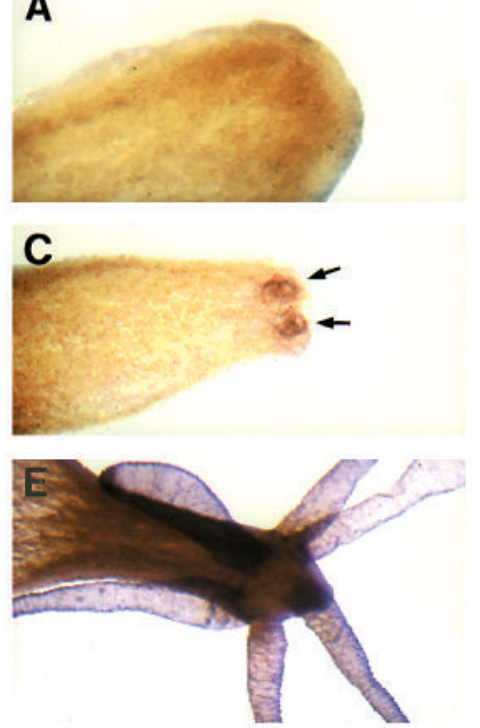

B

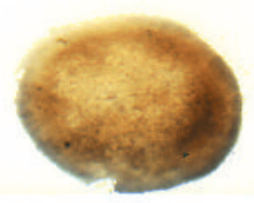

D

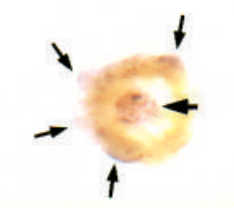

$\mathbf{F}$

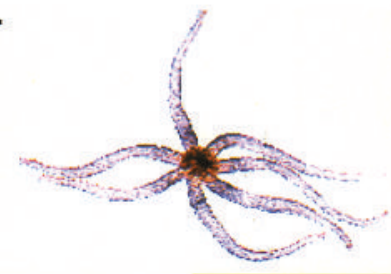

Fig. 7. Activation of expression of $k s 1$ during head regeneration. A/B, 1 day after decapitation. C/D, 2 days after decapitation. Filled arrow points to staining in hypostomal zone. E/F, 4 days after decapitation. $k s l$ expression is indicated by the purple-blue staining along the entire length of the tentacles. The body column (E) and hypostome (F, center) are not stained and appear brownish. A, C and $\mathrm{E}$ are from side. $\mathrm{B}, \mathrm{D}$ and $\mathrm{F}$ are from above. Staining is restricted to tentacle epithelial cells.

leader sequence and several distinct domains of charged amino acid residues (Fig. 4C). The first cluster of basic and acidic amino acids (residues 32-96) shows significant sequence similarity to the second cluster of basic and acidic amino acids (residues 97-147) and may be due to sequence duplication. Several other proteins with highly acidic domains have been described and include nuclear-localized proteins such as nucleolin and cytosolic proteins such as the yeast sec 7 protein. In all these proteins, the acidic domains are thought to serve as structural motifs for interaction with lipids or other proteins (Achstetter et al., 1988). Hydra KS1 appears to be a secreted protein and could be adsorbed on the cell surface by its highly charged amino acid domains. It may be a component of the carbohydrate-rich glycocalyx that covers the surface of hydra polyps.

A monoclonal antibody, $\mathrm{CP} 8$, which specifically recognizes ectodermal epithelial cells in the head (Javois et al., 1986), has been shown to detect a protein localized in granules near the apical surface of ectodermal epithelial cells. Since KS1 contains a leader sequence, it could encode a secretory protein and be located, at least transiently, in cytosolic granules. It is intriguing to speculate that the $k s 1$ gene product is the CP8 antigen.

\section{Position-dependent expression of $k s 1$}

The upper gastric region subjacent to tentacles has previously been defined as the 'tentacle formation zone' (Hobmayer et al., 1990); epithelial cells in this region become committed to battery cell differentiation and are displaced into the tentacles. $k s l$ is expressed at high levels in battery cells as well as in a subpopulation of ectodermal epithelial cells in the tentacle formation zone. $k s 1$-expressing cells in this region are mor-

A

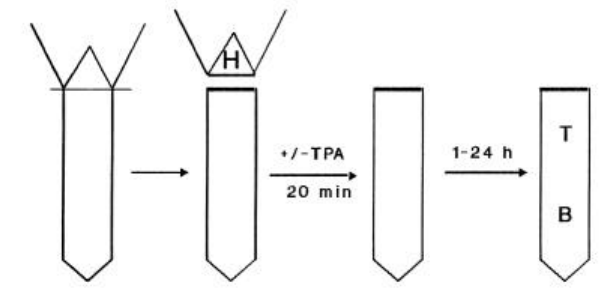

B

$\mathrm{H}$

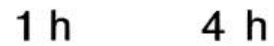

$24 \mathrm{~h}$

ks1

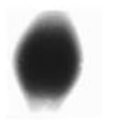

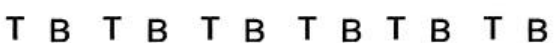

actin

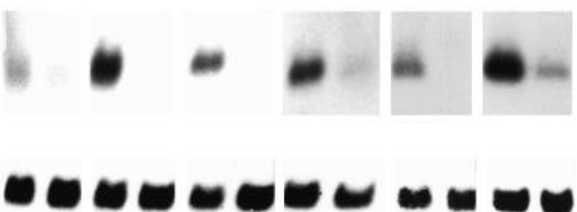

Fig. 8. Effect of TPA on expression of $k s l$ in regenerating polyps. (A) Experimental procedure. (B) Northern blot using $15 \mu \mathrm{g}$ of total RNA and a $k s 1$ cDNA probe. A hydra actin cDNA kindly provided by Hans Bode was used as control to demonstrate equal loading of RNA. T, upper gastric region; B, lower gastric region. -, untreated polyps. + , polyps exposed to $30 \mathrm{nM}$ TPA for 20 minutes.

phologically indistinguishable from $k s l$-negative cells. Thus, $k s 1$ is a very early marker for head-specific differentiation.

During head regeneration, at a stage when tentacles are just beginning to evaginate ( $\mathrm{C}$ and $\mathrm{D}$ in Fig. 7), $k s l$ expression is observed at the most apical tip and in the tentacle buds. At later stages, no expression of $k s l$ is detected in the tip whereas the tentacles are strongly stained. This pattern of $k s l$ expression is strikingly similar to that found previously with the headspecific monoclonal antibody TS-19 (Bode et al., 1988). During head regeneration, TS-19 label first appears at the most apical tip. At later stages, it spreads to the evaginating tentacles and disappears from the apex.

The transient appearance of $k s 1$ and TS19 in the most apical region of regenerating tips is consistent with the view (Bode et al., 1988) that the presumptive hypostome area passes through a stage of 'tentacleness' before final differentiation. This pattern of expression of $k s l$ and TS-19 can be explained by a model proposed recently by Hans Meinhardt (1993). According to Meinhardt's model, tentacle formation is activated at regenerating tips; hypostome formation then locally inhibits tentacle formation and displaces it to the subhypostomal region. $k s l$ could be a suitable molecular probe to dissect the mechanism behind this patterning process.

\section{Hydra head-specification appears to involve a receptor-activated protein kinase $\mathrm{C}$ second messenger pathway}

The northern analysis in Fig. 8 indicates a rapid and a delayed effect of TPA on $k s l$ expression. The rapid effect appears to be due to direct activation of the second messenger system in cells already expressing $k s l$ since TPA treatment simply increases the amount of $k s l$ transcript but does not increase the number of positive cells. This suggests that $k s 1$ expression is 
regulated by a ligand-activated PKC second messenger pathway. The observation that $k s l$ expression is also increased by 4 hours of regeneration in tissue untreated with TPA suggests that the endogenous ligand begins to be produced by this time.

By comparison, the delayed effect, which leads to generation of ectopic $k s 1$-positive cells in the lower body column and requires at least 4 hours to become detectable, appears to be an indirect effect of TPA treatment. We imagine that TPA activates a signaling system for head formation in the body column and that this signal then induces ectopic formation of $k s l$-positive cells.

Two potential cis-regulatory elements are present in the $5^{\prime}$ flanking sequences of $k s l$ and might serve to transmit the TPA signal(s) to the transcriptional machinery. $k s l$ is the first gene in hydra found to be sensitive to TPA; its structure and expression pattern fulfill a prediction that followed from Müller's results (Müller 1989, 1993). Whether other headspecific genes in hydra also have TPA-responsive sequence elements in their $5^{\prime}$ flanking sequences remains to be elucidated. It is interesting to note, however, that Cnox-2, a Hydra homeobox gene thought to be involved in axial patterning, also seems to be regulated by a signal transduction pathway involving PKC (Shenk et al., 1993).

Similar observations have been made in several other developing organisms. PKC has been suggested to participate in controlling differentiation along an embryonic axis in Xenopus (Otte et al., 1988), in determining cell fate in Dictyostelium (Ginsburg and Kimmel, 1989; Peters et al., 1989), and in establishing cell fate in sea urchin embryos (Livingston and Wilt, 1992).

Regardless of the mechanisms by which PKC modulates $k s 1$ expression in Hydra, the results presented in this paper support the hypothesis that signaling mediated by ligand/receptor interactions at the plasma membrane is important in instructing hydra cells to differentiate according to their position in the embryo. Studying $k s l$ expression may now allow us to trace the mechanism necessary for transcriptional activation back to events at the cell surface.

We are grateful to Drs Klaus Gellner and Maria Lopez de Haro for critical discussion of the data, to Dr Michael Sarras and Dr Hans Bode for providing the H.vulgaris cDNA library, to Dr Robert Steele for comments on the manuscript, and to Gabriele Praetzel for excellent technical help. L. M. S. received a postdoctoral fellowship from the Alexander-von-Humboldt foundation, Bonn. Supported by the Deutsche Forschungsgemeinschaft (grants to C. N. D. and T. C. G. B.).

\section{REFERENCES}

Achstetter, T., Franzusoff, A., Field, C. and Schekman, R. (1988). SEC7 encodes an unusual, high molecular weight protein required for membrane traffic from the yeast Golgi apparatus. J. Biol. Chem. 263, 11711-11717.

Angel, P., Imagawa, M., Chiu, R., Stein, B., Imbra, R. J., Rahmsdorf, H., Jonat, C., Herrlich, P. and Karin, M. (1987). Phorbol ester-inducible genes contain a common cis element recognized by a TPA-modulated transacting factor. Cell 49, 729-739.

Berridge, M. J., Downes, C. P. and Hanley, M. R. (1989). Neural and developmental actions of lithium: a unifying hypothesis. Cell 59, 411-419.

Bode, P. M. and Bode, H. R. (1984). Patterning in hydra. In Pattern
Formation. A Primer in Developmental Biology (ed. G. M. Malacinski and S. V. Bryant) London, New York: MacMillan Publishing Co.

Bode, P. M., Awad, T. A., Koizumi, O., Nakashima, Y., Grimmelikhuijzen, C. I. P. and Bode, H. R. (1988). Development of the two-part pattern during regeneration of the head in hydra. Development 102, 223-235.

Bosch, T. C. G., Unger, T. F., Fisher, D. A. and Steele, R. E. (1989). Structure and expression of STK, a src-related gene in the simple metazoan Hydra attenuata. Mol. Cell. Biol. 9, 4141-4151.

Gellner, K., Praetzel, G. and Bosch, T. C. G. (1992). Cloning and expression of a heat-inducible hsp70 gene in two species of Hydra which differ in their stress response. Eur. J. Biochem. 210, 683-691.

Ginsburg, G. and Kimmel, A. R. (1989). Inositol triphosphate and diacylglycerol can differentially modulate gene expression in Dictyostelium. Proc. Natl. Acad. Sci. USA 86, 9332-9336.

Hassel, M., Albert, K. and Hofheinz, S. (1993). Pattern formation in Hydra vulgaris is controlled by lithium-sensitive processes. Dev. Biol. 156, 362371 .

Hobmayer, E., Holstein, T. W. and David, C. N. (1990). Tentacle morphogenesis in hydra. II. Formation of a complex betwen a sensory nerve cell and a battery cell. Development 109, 897-904.

Javois, L. C., Wood, R. W. and Bode, H. R. (1986). Patterning of the head in Hydra as visualized by a monoclonal antibody. I. Budding and regeneration. Dev. Biol. 117, 607-618.

Kurz, E. M., Holstein, T. W., Petri, B. M., Engel, J. and David, C. N. (1991). Mini-collagens in hydra nematocytes. J. Cell Biol. 115, 1159-1169.

Lenhoff, H. M. and Brown, R. D. (1970). Mass culture of hydra: An improved method and its application to other aquatic invertebrates. Lab. Anim. 4, 139154.

Livingston, B. T. and Wilt, F. H. (1992). Phorbol esters alter cell fate during development of sea urchin embryos. J. Cell. Biol. 119, 1641-1648.

Lopez de Haro, M. S., Salgado, L. M., David, C. N. and Bosch, T. C. G. (1994). Hydra tropomyosin TROP1 is expressed in head specific epithelial cells and is a major component of the cytoskeletal structure which anchors nematocytes. J. Cell Sci. 107, 1403-1411.

Meinhardt, H. (1993). A model for pattern formation of hypostome, tentacles, and foot in hydra: how to form structures close to each other, how to form them at a distance. Dev. Biol. 157, 321-333.

Müller, W. A. (1989). Diacylglycerol induced multihead formation in Hydra. Development 105, 309-316.

Müller, W. A. (1993). Pattern control in Hydra: basic experiments and concepts. In Experimental and Theoretical Advances in Biological Pattern Formation (ed. H. G. Othmer), pp 237-253. New York: Plenum Press.

Muraosa, Y. and Shibahara, S. (1993). Identification of a cis-regulatory element and putative trans-acting factors responsible for 12-Otetradecanoylphorbol-13-acetate (TPA)-mediated induction of heme oxygenase expression in myelomonocytic cell lines. Mol. Cell. Biol. 13, 7881-7891.

Nishizuka, Y. (1986). Studies and perspectives of protein kinase C. Science 233, 305-312.

Otte, A. P., Koster, C. H., Snoek, G. T. and Durston, A. J. (1988). Protein kinase C mediates neural induction in Xenopus laevis. Nature 334, 618-620.

Peters, D. J. M., Van Lookeren Campagne, M. M., Van Haastert, P. J. M., Spek, W. and Shaap P. (1989). Lithium ions induce prestalk-associated gene expression and inhibit prespore gene expression in Dictyostelium discoideum. J. Cell Sci. 93, 205-210.

Sambrook, J., Fritsch, D. M. and Maniatis, T. (1989). Molecular Cloning: a Laboratory Manual. 2nd edition. Cold Spring Harbor Laboratory Press.

Shenk, M. A., Bode, H. R. and Steele, R. E. (1993). Expression of Cnox-2, a $\mathrm{HOM} / \mathrm{HOX}$ homeobox gene in hydra, is correlated with axial pattern formation. Development 117, 657-667.

Shenk, M. A. and Steele, R. E. (1993). A molecular snapshot of the metazoan 'Eve'. Trends Biochem. Sci. 18, 459-463.

Summerbell D., Smith, J. C. and Maden, M. (1991). The molecular basis of positional information. In Vivo 5, 457-472.

Von Heijne, G. (1983). Pattern of amino acids near signal - sequence cleavage sites. Eur. J. Biochem. 133, 17-21.

Wolpert, L. (1969). Positional information and the spatial pattern of cellular differentiation. J. Theoret. Biol. 25, 1-47.

Wolpert, L., Hornbruch, A. and Clarke, M. R. B. (1974). Positional information and positional signaling in hydra. Amer. Zool. 14, 647-663.

(Accepted 3 June 1994) 